\title{
Software Defined Radio Based Readout of Microwave SQUID Multiplexed Metallic Magnetic Calorimeter Arrays
}

\author{
Oliver Sander, Nick Karcher, Oliver Kroemer, Marc Weber \\ IPE, Karlsruhe Institute of Technology (KIT), Karlsruhe, Germany \\ E-mail: sander@kit.edu, karcher@kit.edu, oliver.kroemer@kit.edu, \\ marc.weber@kit.edu
}

\section{Sebastian Kempf, Mathias Wegner, Christian Enss \\ KIP, University Heidelberg, Heidelberg, Germany \\ E-mail: sebastian.kempfakip.uni-heidelberg.de, \\ mathias.wegnerastud.uni-heidelberg.de, \\ christian.enssakip.uni-heidelberg.de}

\begin{abstract}
Metallic magnetic calorimeters (MMCs) are cryogenic detectors that offer an excellent energy resolution, a signal rise time of below $100 \mathrm{~ns}$, a high dynamic range and almost optimal linearity. MMCs are of high interest for many experiments, such as dark matter detection or direct neutrino mass investigations. Since larger detector arrays are read out at GHz-Frequencies, and each single pixel offers a fast rise time, a complex analog and digital readout system is required. This contribution will give an introduction to MMC technology in combination with the implemented readout electronics and the current status of the present readout architecture.
\end{abstract}

Topical Workshop on Electronics for Particle Physics

11 - 14 September 2017

Santa Cruz, California 


\section{Metallic Magnetic Calorimeters}

Metallic Magnetic Calorimeters (MMCs) [1] are calorimetric low-temperature particle detectors that are currently strongly advancing the state-of-the-art in energy-dispersive single particle detection. MMCs are typically operated at temperatures well below $100 \mathrm{mK}$ and make use of a metallic, paramagnetic temperature sensor to transduce the temperature rise of the detector upon the absorption of an energetic particle into a change of magnetic flux (see Figure 1). The latter can be precisely measured using a superconducting quantum interference device (SQUID). This outstanding interplay between a high-sensitivity magnetic thermometer and a near quantum-limited amplifier results in a very fast signal rise time reaching values below $100 \mathrm{~ns}$. Furthermore, they achieve an excellent energy resolution which is competitive to the resolving power of wavelengthdispersive crystal spectrometers, a large energy dynamic range, a high quantum efficiency as well as an almost ideal linear detector response. For this reason, a growing number of groups located all over the world are developing MMC arrays of various sizes which range from a few to several thousand pixels. These arrays are routinely used in a variety of applications and often appear to be a key technology for measurements that require high-resolution and wideband energy-resolving detectors. MMCs have already proven to be eminently suited detectors for various applications such as high resolution X-ray spectroscopy [2], nuclear forensics [3], radiation metrology [4] or direct neutrino mass investigation. In particular, the "Electron capture in Holmium-163 experiment" (ECHo) [5, 6], which aims to investigate the electron neutrino mass in the sub-eV/c $\mathrm{c}^{2}$ range by performing a calorimetric measurement of the ${ }^{163} \mathrm{Ho}$ electron capture spectrum, requires the acquisition of a high-resolution and high-statistics spectrum with up to $10^{14}$ decays. Due to constraints on the activity per pixel to limit internal background caused by a pile-up of detector events, large detector arrays with up to $10^{6}$ detectors running in parallel are required to acquire such a high statistics spectrum in finite time.

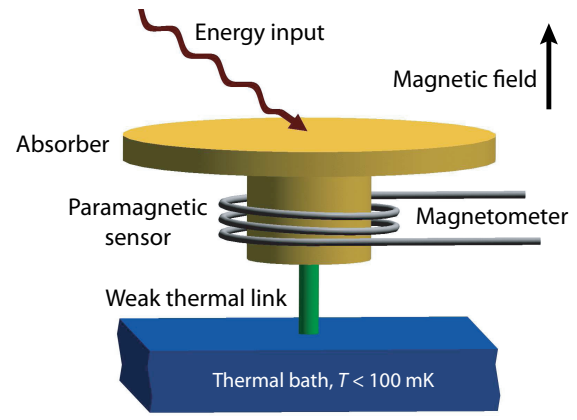

Figure 1: Schematic drawing of an MMC

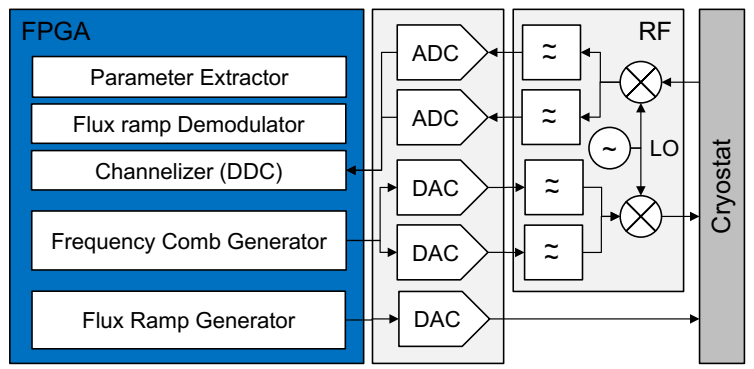

Figure 2: Overview of the SDR hardware

\section{Microwave SQUID Multiplexing}

An efficient readout of large MMC arrays can be achieved through Microwave SQUID multiplexing [7-9]. It requires three different components or techniques for proper operation: (1) The actual cryogenic multiplexer which modulates the detector signals onto different carrier signals and combines them into a single readout line, (2) a room temperature readout electronics for operating 
the multiplexer as well as (3) a method for linearizing the multiplexer response to maintain the intrinsic linearity of MMCs.

Each channel of a microwave SQUID multiplexer consists of a non-hysteretic, unshunted, current-sensing rf-SQUID [7]. The SQUID is inductively coupled to a load inductor terminating a superconducting $\lambda / 4$-transmission line resonator having a resonance frequency in the $\mathrm{GHz}$ range. Due to the mutual interaction between the SQUID and the load inductor, the circuit's resonance frequency gets magnetic flux dependent and is shifted as the magnetic flux introduced to the SQUID loop changes. For simultaneous readout of $N$ detectors, $N$ resonance circuits, each with a unique resonance frequency, are capacitively coupled to a common transmission line. This arrangement allows the measurement of the actual resonance frequencies of all channels by injecting a microwave frequency comb into the common transmission line where. All individual comb tones are set with respect to the undisturbed resonance frequency of the different channels. The sensor signals modulate amplitude or phase of each transmitted tone.

A straightforward technique to generate the microwave frequency comb as well as to determine the amplitude or phase of each transmitted carrier signal is software defined radio (SDR). The frequency comb is created in the baseband between $0 \mathrm{~Hz}$ and several $100 \mathrm{MHz}$ using two fast digital-to-analog converters (DACs). The frequency comb is then up-converted into the frequency band of the SQUID multiplexer using an $I / Q$ mixer to match the resonance frequencies of the resonators. After injecting the frequency comb into the cryostat and passing the multiplexer, the modulated frequency comb is mixed into the baseband again using an $I / Q$ down-mixer. Both mixers are driven by the same local oscillator signal. The resulting in in-phase and quadrature baseband signals are digitized by two fast analog-to-digital converters (ADCs). The subsequent signal processing, i.e. the separation of the frequency-comb into the different carrier signals, the determination of amplitude and phase of each carrier signal as well as the determination of the characteristic parameters of each detector signal, is then performed by using a field programmable gate array (FPGA).

Due to the periodicity of the SQUID characteristics, the output signal of a microwave SQUID multiplexer is non-linear. Consequently, additional measures are required to linearize the output signal. Flux ramp modulation [10] is a very suited approach that relies on simultaneously applying a fast and periodical sawtooth-shaped magnetic flux signal to all SQUIDs. The quasi-static detector signal within one flux ramp period adds a constant magnetic flux offset to the SQUID. The phase difference between the SQUID characteristics with and without additional flux offset due to the detector is directly proportional to the detector signal and can be used as a linearized output signal.

\section{Software Defined Radio Architecture}

The current SDR concept of the ECHo experiment in its $100 \mathrm{kBq}$ stage utilizes 15 boards, each covering $4 \mathrm{GHz}$. With a channel spacing $10 \mathrm{MHz}$ this results in 6000 channels. As shown in Figure 2, the SDR architecture can be separated into three sections RF, digitization and digital processing. Latter is performed by a heterogeneous Xilinx Zynq Ultrascale+ FPGA [11], which is a combination of a Kintex Ultrascale+ FPGA fabric with a powerful ARM A53 based quad-core processor system. The processors perform slow control including calibration and data transmission to the storage backend. All significant processing steps depicted in Figure 2 run on the FPGA. 
The frequency-comb generator store and plays precomputed waveforms and therefore is a simple arbitrary waveform generator. The flux-ramp is generated in the same way. On receiver site, the samples coming from the ADC are at first processed in a channelizer, which extracts the various channels. Afterwards, the flux-ramp is demodulated for each channel. Then the detector signals are available, and the relevant parameters can be obtained in the last stage. After all relevant event parameters were extracted, the data will be hand over to the software layers. These pack the data and transmit it to the backend storage system.

Digitization is based on an 1 GSPS, two-channel, 16 bit analog-to-digital converter with an JESD204b interface. The converter allows sampling $1 \mathrm{GHz}$ of complex analog bandwidth. On the transmission side a corresponding 1 GSPS, four channel, 16 bit digital-to-analog converter will be used to transform I/Q samples into a complex baseband signal. Covering a $4 \mathrm{GHz}$ range requires five ADC and three DAC chips, assuming each has $800 \mathrm{MHz}$ bandwidth. Latter generates some overlap which is beneficial for the RF filter design. Figure 3 depicts the transmission and reception side. For transmission, the complex baseband signal is filtered and up-mixed after the DA conversion. On the receiver side, the signal is down-mixed, then the complex baseband signal is amplified and filtered in various stages before the AD conversion. The mixers get the carrier from a local clock chip. Figure 4 depicts the whole board that comprises five of the transceiver stages each having a different local oscillator frequency.

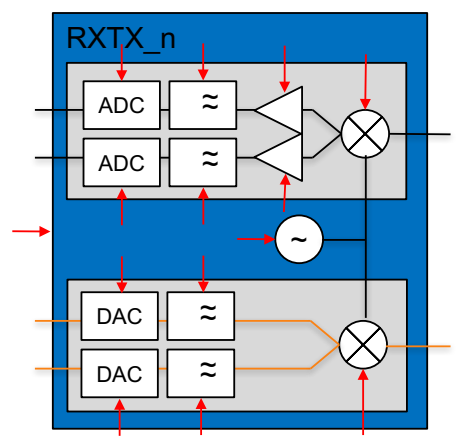

Figure 3: Detailed view on RFboard

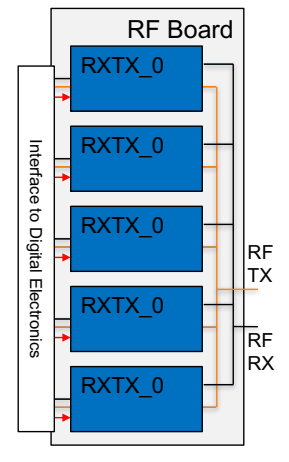

Figure 4: Concept of the RF-board

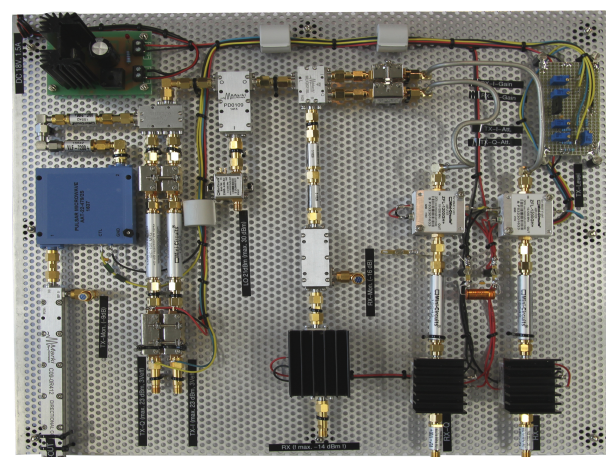

Figure 5: Advanced RF-Frontend

\section{RF-Measurements}

Before building the ECHo RF board and after initial measurements [12] a prototypical highfrequency setup was assembled, to achieve a proper signal conditioning (see Figure 5). In send direction, two adjustable attenuators were introduced to set a suitable and balanced input power before mixing with the frequency of the local oscillator. Marki Microwave MLIQ0218L mixers are used in the up- and down-converting stage. Also filters with a cut-off frequency of $105 \mathrm{MHz}$ were chosen for DA reconstruction and against aliasing. On receiver side a $50 \mathrm{~dB}$ amplifier was used before mixing. Afterwards, an adjustable amplifier section was integrated to balance and adjust the signal level before AD conversion.

After AD conversion by a two-channel ADS54J69 500 MS/s ADC the digitized signal is then processed in an FPGA firmware on a Xilinx VC707 board (see Figure 6). In a first section, the 
signals of two resonators are channelized by a digital down conversion stage. This stage multiplies the incoming signal with a complex frequency to shift the signals in frequency. Afterwards, they are low-pass filtered and decimated to remove the remaining signal from other channels. During the decimation, the data rate is reduced from $2 \mathrm{~GB} / \mathrm{s}$ to $125 \mathrm{MB} / \mathrm{s}$ for two channels. The demodulated Signals are packed in UDP packages and transmitted to a control PC via $10 \mathrm{GBit} / \mathrm{s}$ Ethernet. The flux ramp modulation can be seen directly in the data stream (see Figure 7). To generate the required stimulation frequencies a playback module reads samples from a block RAM at $500 \mathrm{MS} / \mathrm{s}$. The samples are interpolated, and DA converted at $1 \mathrm{GS} / \mathrm{s}$ by a DAC39J84 chip.

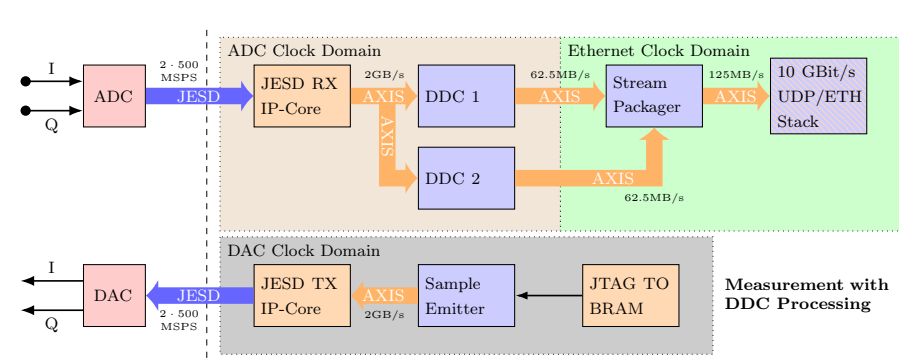

Figure 6: DDC Architecture

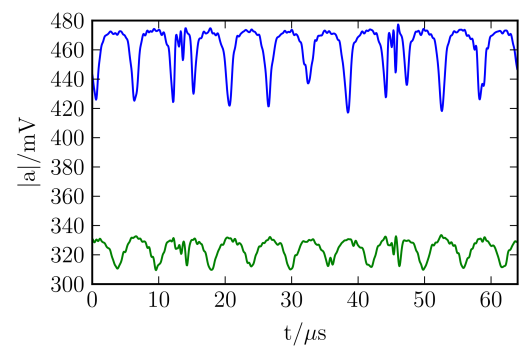

Figure 7: Measurement with two resonator channels

\section{Conclusion}

The relatively complex readout of MMC arrays can be tackled using software defined radios. This paper gave a brief introduction into MMCs, microwave SQUID multiplexing as readout technique and presented ECHo's SDR concept. Additionally, some results of our RF measurements were described. First demonstrators [12] proofed the feasibility of this concept and evaluation of wide-band RF setups show promising results. As a result, the ECHo-100k SDR will be developed based on these achievements.

\section{References}

[1] A. Fleischmann et al. Metallic Magnetic Calorimeters. pp. 151-216. Springer, 2005.

[2] B. Simmnacher et al. Microelectronics Reliability. 43(9):1675-1680, 2003.

[3] S. Okada et al. Journal of Low Temperature Physics. 176(5):1015-1021, 2014.

[4] K. Mitsuda et al. Journal of Low Temperature Physics. 167(5):795-802, 2012.

[5] L. Gastaldo et al. Journal of Low Temperature Physics. 176(5):876-884, 2014.

[6] L. Gastaldo et al. The European Physical Journal Special Topics. 226(8):1623-1694, 2017.

[7] J. A. B. Mates et al. Applied Physics Letters. 92(2):023514, 2008.

[8] S. Kempf et al. Superconductor Science and Technology. 30(6):065002, 2017.

[9] S. Kempf et al. AIP Advances. 7(1):015007, 2017.

[10] J. A. B. Mates et al. J. Low. Temp. Phys. 167(5-6):707-712, 2012.

[11] Xilinx. DS891, Zynq UltraScale+ MPSoC Data Sheet: Overview. 2017.

[12] M. Wegner et al. Microwave SQUID Multiplexing of Metallic Magnetic Calorimeters: Status of Multiplexer performance and room-temperature readout electronics development (LTD 2017). 2017. 